\title{
Glomerular filtration rate and initiation of dialysis
}

\author{
Vianda S. Stel PhD, Kitty J. Jager MD PhD \\ See related research article by Clark and colleagues, page 47
}

Competing interests: None declared.

This article was solicited and has not been peer reviewed.

Correspondence to: Dr. Vianda S. Stel, v.s.stel@amc.uva.nl

CMAJ 2011. DOI:10.1503 /cmaj.101636
I $\mathrm{n}$ this issue, Clark and colleagues ${ }^{1}$ present data showing a relationship between higher estimated glomerular filtration rate at the start of hemodialysis and mortality. Their results confirm the findings of previous observational studies. In this commentary, we focus on what can be learned from publications so far about interpreting this counterintuitive finding.

In their study, Clark and colleagues ${ }^{1}$ estimated glomerular filtration rate by the abbreviated modified diet in renal disease formula in a cohort of 25901 adult patients starting hemodialysis obtained from the Canadian Organ Replacement Register. The unadjusted hazard ratio for those with early initiation of hemodialysis (estimated glomerular filtration rate $>10.5$ $\mathrm{mL} / \mathrm{min}$ per $1.73 \mathrm{~m}^{2}$ ) relative to those with late initiation of hemodialysis (estimated glomerular filtration rate $\leq 10.5 \mathrm{~mL} / \mathrm{min}$ per $1.73 \mathrm{~m}^{2}$ ) was 1.48 (95\% confidence interval [CI] 1.43-1.54). The hazard ratio declined to 1.18 (95\% CI 1.131.23) after adjustment for demographic characteristics, serum albumin level, type of vascular access, comorbidities and transplant status. The association between higher estimated glomerular filtration rate and increased risk of death could therefore not be fully explained by differences in baseline characteristics.

Similar associations have been observed for patients starting dialysis across the globe..$^{2-8}$ However, it is evident that when it comes to assessing the effect of interventions, observational studies have certain limitations. First,

\footnotetext{
- KEY POINTS

- Study results to date suggest either no effect of starting dialysis at higher levels of estimated glomerular filtration rate or an increased risk of death.

- Those studies applied widely used equations for glomerular filtration rate that have not been validated in patients with rates below $20 \mathrm{~mL} / \mathrm{min}$.

- Nephrologists may also use criteria other than estimated or measured glomerular filtration rate in the decision to start dialysis, but it is unclear which criteria they use.

- Research is needed to determine if starting dialysis at higher levels of renal function is harmful.
}

patients for whom dialysis is started at higher estimated glomerular filtration rate are known to suffer more from comorbidity and severe malnutrition and may therefore have a higher risk of death than those whose dialysis is started at lower estimated glomerular filtration rate. This represents confounding by indication, and even after adjustment for known confounders, there is still the possibility of confounding by unmeasured confounders. Second, in most observational studies, data have been available only from point of initiation of dialysis. In this situation, the results may be affected by lead-time bias (i.e., favouring survival of groups with high estimated glomerular filtration rate, because these groups enter the study earlier in the disease process and therefore "survive" for a longer period) and survivor bias (i.e., favouring survival of groups with low estimated glomerular filtration rate, as these patients may have selected themselves out as survivors). Two previous studies avoided leadtime and survivor biases by enrolling patients when their estimated glomerular filtration rate dropped below a particular threshold. ${ }^{3.8}$ In one of the studies, the mortality rate was higher among patients who started dialysis at higher estimated glomerular filtration rate, ${ }^{3}$ whereas the other study found no difference. ${ }^{8}$

Recently, the first randomized controlled trial (RCT) on this topic, the Initiating Dialysis Early and Late (IDEAL) study, was performed in Australia and New Zealand. ${ }^{9}$ The randomization prevented confounding by indication. Also, because observation of patients in both arms started from the same time point in the disease process, neither survivor bias nor lead-time bias could affect the results. The IDEAL study showed no beneficial effect in terms of survival of patients randomly assigned to "early" initiation of dialysis (estimated glomerular filtration rate 10-14 $\mathrm{mL} / \mathrm{min}$ per $1.73 \mathrm{~m}^{2}$ ) relative to those assigned to "late" initiation (estimated glomerular filtration rate $5-7 \mathrm{~mL} / \mathrm{min}$ per $1.73 \mathrm{~m}^{2}$ ). However, in the late-start group, $76 \%$ of the patients needed dialysis because of uremic symptoms when their

All editorial matter in CMAJ represents the opinions of the authors and not necessarily those of the Canadian Medical Association. 
estimated glomerular filtration rate was far above $5-7 \mathrm{~mL} / \mathrm{min}$ per $1.73 \mathrm{~m}^{2}$. In fact, the mean estimated glomerular filtration rate at the start of dialysis was $9.0 \mathrm{~mL} / \mathrm{min}$ per $1.73 \mathrm{~m}^{2}$ in the earlystart group and $7.2 \mathrm{~mL} / \mathrm{min}$ per $1.73 \mathrm{~m}^{2}$ in the late-start group. Therefore, the results suggested that the decision to start dialysis was guided not only by the estimated glomerular filtration rate, but also by the patient's clinical condition. Furthermore, for a number of reasons, the participants in the IDEAL study may not have been typical of dialysis patients seen in everyday clinical practice in other countries.

The observational studies and the recently performed RCT leave the impression that in terms of patient survival adjusted for confounders, either it does not make any difference if patients start at a higher level of renal function or starting at higher levels may be harmful. The latter would be worrying, because registry data have shown a trend for increasing mean estimated glomerular filtration rate at initiation of dialysis. ${ }^{1}$ However, for a number of reasons, the results of studies performed to date should be interpreted with caution.

All of the studies used creatinine-based estimates of glomerular filtration rate, calculated by either the modified diet in renal disease equation or the Cockcroft and Gault equation. The problem with using these equations for this specific purpose is that they have never been validated in patients with glomerular filtration rate below 20 $\mathrm{mL} / \mathrm{min}$. Interestingly, Grootendorst and colleagues ${ }^{10}$ showed a low correlation $(0.51)$ between glomerular filtration rate at the start of dialysis as estimated by the abbreviated modified diet in renal disease equation and glomerular filtration rate measured as the mean of urea and creatinine clearance based on 24-hour urine collection. In addition, higher estimated glomerular filtration rate at the start of dialysis was associated with a higher risk of death, but measured glomerular filtration rate was not significantly associated with mortality. Grootendorst and colleagues ${ }^{10}$ concluded that the modified diet in renal disease equation is unsuitable for estimating glomerular filtration rate for patients who are starting dialysis. An important reason might be that in the presence of low glomerular filtration, the patient's muscle mass will be a relatively more important determinant of plasma creatinine levels than glomerular filtration rate, leading to too-high estimates of the rate in patients with low muscle mass.

Furthermore, in accord with the RCT described above, the observational studies have suggested that nephrologists use criteria other than glomerular filtration rate (estimated or measured) in the decision to start dialysis. Currently, it is unclear which criteria they use to determine the "best" time to start dialysis and to what extent these criteria vary from one nephrologist to another. In addition, there is a lack of evidence as to which criteria should be considered the most important in relation to patient outcomes such as survival and quality of life.

Clark and colleagues ${ }^{1}$ have correctly left open the possibility that starting hemodialysis early may actually be harmful. They refer to previously published studies ${ }^{11,12}$ presenting the interesting hypothesis that sudden cardiac death, a common cause of death among patients undergoing dialysis, may be precipitated by the dialysis procedure itself. This hypothesis is based on the observation that ultrafiltration from the dialysis may increase the patient's risk for hypotension, especially at higher residual renal function. These and other points remain to be addressed in future research.

\section{References}

1. Clark WF, Na Y, Rosansky SJ, et al. Association between estimated glomerular filtration rate at initiation of dialysis and mortality. CMAJ 2011;183:47-53.

2. Beddhu S, Samore MH, Roberts MS, et al. Impact of timing of initiation of dialysis on mortality. J Am Soc Nephrol 2003;14: 2305-12.

3. Evans M, Tettamanti G, Nyrén O, et al. No survival benefit from early-start dialysis in a population-based, inception cohort study of Swedish patients with chronic kidney disease. J Intern Med 2010 Aug. 24 [Epub ahead of print].

4. Hwang SJ, Yang WC, Lin MY, et al. Impact of the clinical conditions at dialysis initiation on mortality in incident haemodialysis patients: a national cohort study in Taiwan. Nephrol Dial Transplant 2010;25:2616-24.

5. Kazmi WH, Gilbertson DT, Obrador GT, et al. Effect of comorbidity on the increased mortality associated with early initiation of dialysis. Am J Kidney Dis 2005;46:887-96.

6. Lassalle M, Labeeuw M, Frimat L, et al. Age and comorbidity may explain the paradoxical association of an early dialysis start with poor survival. Kidney Int 2010;77:700-7.

7. Stel VS, Dekker FW, Ansell D, et al. Residual renal function at the start of dialysis and clinical outcomes. Nephrol Dial Transplant 2009;24:3175-82.

8. Traynor JP, Simpson K, Geddes CC, et al. Early initiation of dialysis fails to prolong survival in patients with end-stage renal failure. J Am Soc Nephrol 2002;13:2125-32.

9. Cooper BA, Branley P, Bulfone L, et al. A randomized, controlled trial of early versus late initiation of dialysis. $N$ Engl J Med 2010;363:609-19.

10. Grootendorst DC, Michels WM, Richardson J, et al. The MDRD formula does not reflect GFR in ESRD patients. Nephrol Dial Transplant 2010 Nov. 5 [Epub ahead of print]

11. McIntyre CW, Stunning M, Ischemia HD, et al. Effects of hemodialysis on cardiac function. Kidney Int 2009;76:371-5.

12. Bleyer AJ, Russell GB, Satko SG. Sudden and cardiac death rates in hemodialysis patients. Kidney Int 1999;55:1553-9.

Affiliations: Vianda S. Stel and Kitty J. Jager are with the Registry of the European Renal Association - European Dialysis and Transplant Association, Department of Medical Informatics, Academic Medical Center, University of Amsterdam, Amsterdam, the Netherlands. 